\title{
Characterization of Spatial Frequency Channels Underlying Disparity Sensitivity by Factor Analysis of Population Data
}

\author{
Alexandre Reynaud * and Robert F. Hess \\ McGill Vision Research, Department of Ophthalmology, McGill University, Montreal, QC, Canada
}

It has been suggested that at least two mechanisms mediate disparity processing, one for coarse and one for fine disparities. Here we analyze individual differences in our previously measured normative dataset on the disparity sensitivity as a function of spatial frequency of 61 observers to assess the tuning of the spatial frequency channels underlying disparity sensitivity for oblique corrugations (Reynaud et al., 2015). Inter-correlations and factor analysis of the population data revealed two spatial frequency channels for disparity sensitivity: one tuned to high spatial frequencies and one tuned to low spatial frequencies. Our results confirm that disparity is encoded by spatial frequency channels of different sensitivities tuned to different ranges of corrugation frequencies.

OPEN ACCESS

Edited by:

Hedva Spitzer,

Tel Aviv University, Israel

Reviewed by:

John E. Lewis,

University of Ottawa, Canada

Hagit Hel-Or,

University of Haifa, Israel

Ronen Segev,

Ben-Gurion University of the Negev,

Beersheba, Israel

*Correspondence:

Alexandre Reynaud

alexandre.reynaud@mail.mcgill.ca

Received: 15 February 2017 Accepted: 28 June 2017

Published: 11 July 2017

Citation:

Reynaud A and Hess RF (2017)

Characterization of Spatial Frequency

Channels Underlying Disparity

Sensitivity by Factor Analysis of

Population Data.

Front. Comput. Neurosci. 11:63.

doi: 10.3389/fncom.2017.00063
Keywords: disparity sensitivity, qDSF, binocular vision, stereopsis, individual differences, factor analysis

\section{INTRODUCTION}

The visual system utilizes the displacement or disparity in the two images seen by the two eyes to compute the depth of objects. In terms of the underlying mechanisms, Pulliam (1982) first suggested that there were two global disparity mechanisms, one tuned to low spatial frequencies involving coarse disparities and one tuned to high spatial frequencies involving fine disparities. Yang and Blake (1991) also argued for only two spatial frequency channels for disparity processing and their model was later refined by Tyler et al. (1994). Additional evidence for two spatial frequency channels subserving disparity processing comes from the work of Norcia et al. (1985); Wilcox and Allison (2009); Witz et al. (2014). However, other studies suggest a multiple channels model (Julesz and Miller, 1975; Glennerster and Parker, 1997; Serrano-Pedraza et al., 2013).

Assessing the tuning of these channels has been of great importance for mechanistic models of stereo computer vision (Marr and Poggio, 1979; Nishihara, 1984; Quam, 1987; Rohaly and Wilson, 1993). These can be used to map different scales of matching in hierarchical structures (Nishihara, 1984; Quam, 1987) with, for instance, coarse-to-fine constraints (Rohaly and Wilson, 1993). In robotic vision, these tuning properties can be used to calibrate cameras (Tsai, 1986) and vergence algorithms (Piater et al., 1999; Lonini et al., 2013).

While most studies have used masking paradigms to characterize spatial frequency channels for stereopsis (Julesz and Miller, 1975; Yang and Blake, 1991; Shioiri et al., 1994; Tyler et al., 1994; Glennerster and Parker, 1997; Prince et al., 1998; Serrano-Pedraza et al., 2013), another possibility comes from factor analysis of population data (Read et al., 2016). The individual differences are then treated as systematic and meaningful, reflecting the true variability of underlying mechanisms rather than random noise (Peterzell, 2016). Identifying the sources of variability within the population will inform on the common processing mechanisms. Therefore, spatial and temporal 
frequency channels can be characterized by analyzing individual differences and correlations. The rationale is that the correlation in detection thresholds for pairs of stimuli should be higher for stimuli detected by the same mechanism than for stimuli detected by different mechanisms (Owsley et al., 1983; Sekuler et al., 1984; Billock and Harding, 1996). Hence by looking at the inter-correlations between individuals' sensitivity at neighboring frequencies, one is able to determine the presence of frequency channels (Mayer et al., 1995; Billock and Harding, 1996; Peterzell and Teller, 2000; Simpson and McFadden, 2005; Rosli et al., 2009). Therefore, a factor analysis of the dataset consisting of a principal component analysis (PCA) and a rotation of the factors in order to determine a simple structure can characterize the tuning curves of the channels (Simpson and McFadden, 2005). Using factor analytics within the population sensitivities Peterzell and Teller $(1996,2000)$ assessed spatial frequency channels tuning for luminance and color contrast sensitivities. Here we use similar methods to analyze individual differences in our previously measured normative dataset on disparity sensitivity as a function of spatial frequency for oblique corrugations of 61 observers (Figure 1; Reynaud et al., 2015) in order to assess the spatial frequency tuning of the underlying disparity channels.

\section{METHODS}

In this paper, we analyze the normative dataset for the disparity sensitivity as a function of spatial frequency of 61 observers $(25$ males, 36 females, mean age 26 years, $\pm 5.7 \mathrm{SD}$, with normal or corrected to normal-visual acuity) we measured previously using the quick Disparity Sensitivity Function ( $q D S F$, Reynaud et al., 2015), a method adapted from the quick Contrast Sensitivity Function ( $q$ CSF, Lesmes et al., 2010).

The stimuli used in this dataset were stereograms composed of spatially filtered 2-D fractal noise carriers with oblique $\left(45^{\circ}\right.$ or $135^{\circ}$ ) sinusoidal corrugations at $0.24,0.33,0.46,0.64,0.89$, $1.23,1.72$, and $2.39 \mathrm{c} / \mathrm{d}$. The spatial frequency of the carrier was 4 times the spatial frequency of the corrugation (see Reynaud et al., 2015). Disparity was modulated and the subjects' task was to identify the orientation of the corrugation in depth $\left(45^{\circ}\right.$ or $135^{\circ}$ ) in a single-interval identification task to measure the disparity detection threshold. Stimuli were displayed on a passive wide 23" 3D-Ready LED monitor ViewSonic V3D231, viewed with polarized 3D glasses at $70 \mathrm{~cm}$, in a dim-lit room. Measured individual disparity sensitivity functions as a function of spatial frequency and their average are reproduced in Figure 1. Analysis was performed with Matlab R2016a (The MathWorks). The hierarchical clustering analysis was specifically performed with the statistics and machine learning toolboxes functions.

\section{RESULTS}

The average disparity sensitivity peaks are in the high spatial frequency range, around $1.2 \mathrm{c} / \mathrm{d}$. However, we can observe a large variability in the individual sensitivities: some showing a low-pass, band-pass or high-pass profiles (Figure 1). Hence a factor analysis of these sensitivities might provide insight into the common mechanisms mediating them.

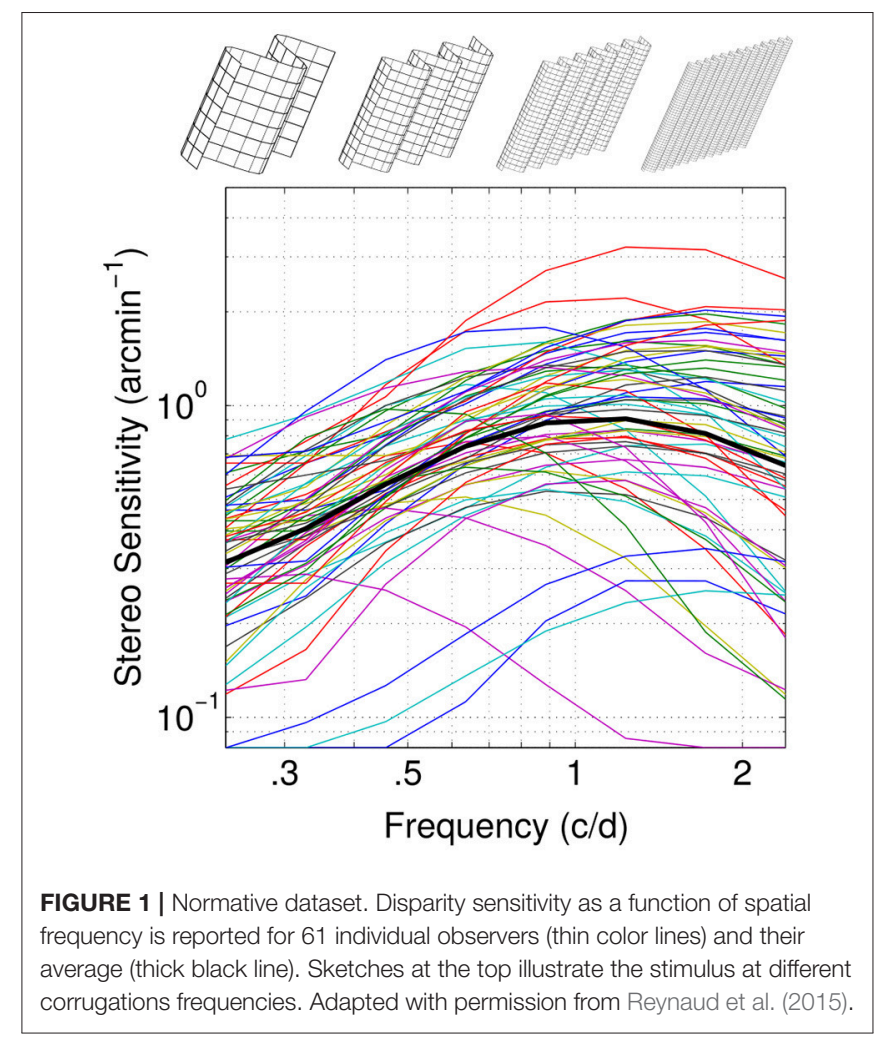

Figure 2 represents the scatterplot matrix of inter-correlations (Peterzell, 2016) for log-disparity sensitivity of all 61 observers. In each cell within the figure, the scatterplot represent the intercorrelation of the log-disparity sensitivity of all observers at one frequency (frequency indicated on the diagonal in the same row) as a function of their sensitivity at another frequency (frequency indicated on the diagonal in the same column) are depicted. For instance, in the bottom-left cell, the log-disparity sensitivity of each observer at $0.24 \mathrm{c} / \mathrm{d}$ is plotted pairwise against its $\log$-disparity sensitivity at $2.39 \mathrm{c} / \mathrm{d}$. Then the coefficient of determination $R^{2}$ between the two frequencies is computed. Two regions of high inter-correlations $\left(R^{2}>0.5\right)$ at low spatial frequency (green) and high spatial frequency (blue) appear along the diagonal.

These two regions are supported by the hierarchical clustering analysis of the log-disparity sensitivity at all spatial frequencies. The pairwise distance between observations was calculated as one minus the sample linear correlation between observations and the hierarchical cluster tree was computed with the average distance. The resulting dendrogram is represented at the right of the inter-correlation matrix, with each spatial frequency being the leaves. Nevertheless, we can note that different distance measures and different linkage procedures can result in relatively different final clusters, some grouping the 3 lowest and 5 highest frequencies for instance. The two cluster branches whose linkage is less than the default $70 \%$ are represented in blue and green. As for the first qualitative approach, these two groups suggest the presence of two spatial frequency channels for disparity sensitivity, which might correspond to the coarse and fine disparity channels. 


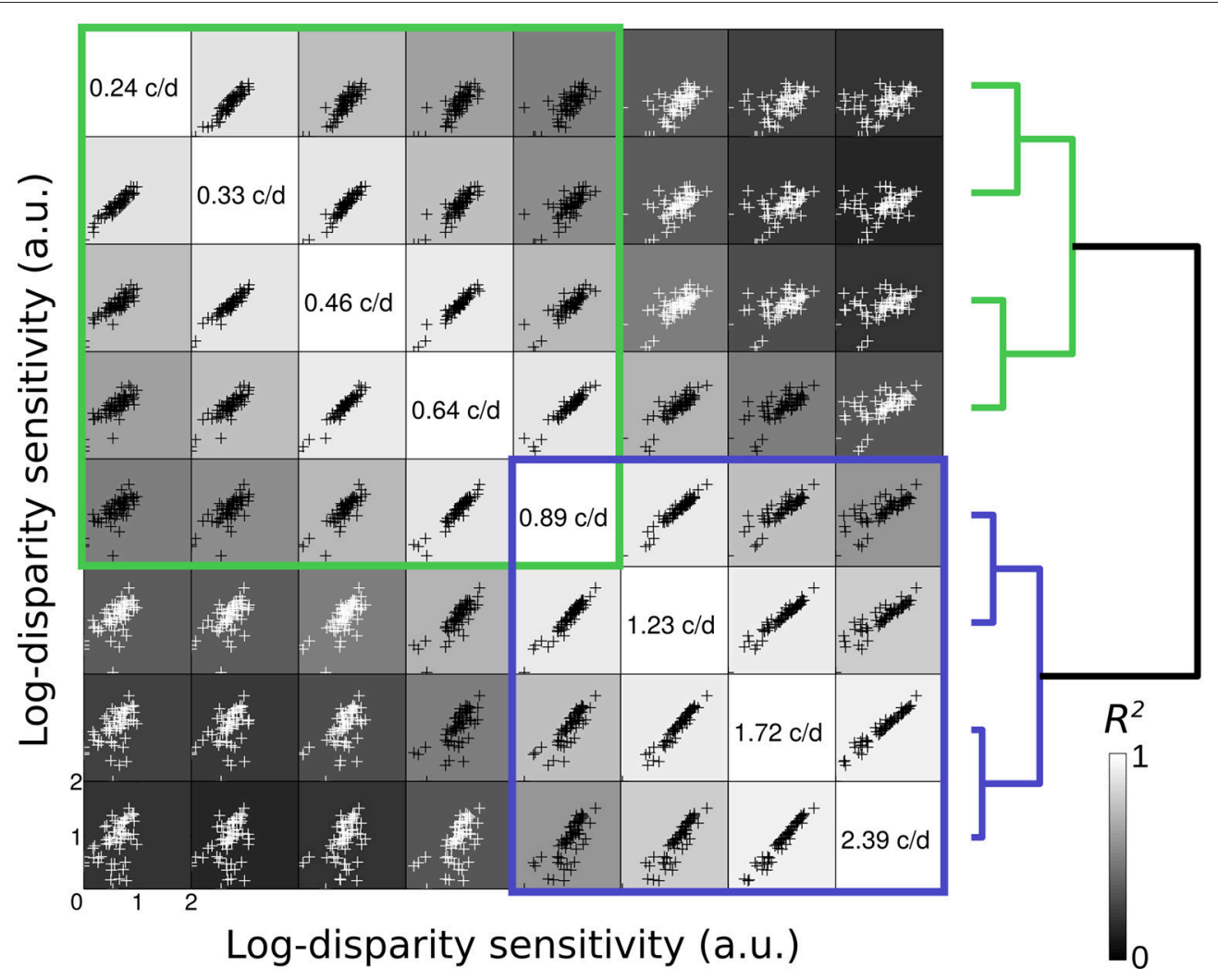

FIGURE 2 | Scatterplot matrix of inter-correlations. In each cell, the scatterplot represent the inter-correlation of the log-disparity sensitivity (arbitrary units) of all 61 observer at one frequency (frequency indicated on the diagonal in the same row) as a function of their sensitivity at another frequency (frequency indicated on the diagonal in the same column). The shade of the background in each cell indicates the value of the coefficient of determination $R^{2}$ between the two frequencies (from black $=0$ to white $=1$ ). Black datapoints indicate $R^{2}>0.5$ and white datapoints $R^{2}<0.5$. Blue and green squares highlight regions of high inter-correlations. On the right is represented the classification dendrogram of the spatial frequencies. The pairwise distance was calculated as one minus the sample linear correlation between observations and the hierarchical cluster tree was computed with the average distance.

In order to determine the precise tuning of these channels, we performed a factor analysis on the dataset. If we decompose the full dataset with a principal component analysis (PCA), we obtain the components shown in Figure 3A, with a percentage of explained variance (calculated from the eigenvalues of the PCA) associated with each component reported in the scree plot Figure 3B.

The first component has the shape of the average sensitivity (see Figure 1). The two first components (blue and green) explain more than $91 \%$ of the variance and the elbow of the scree plot occurs between the second and third components (Figure 3B). As we previously identified two regions of high inter-correlations and that this percentage of explained variance is considered enough to accurately describe the data (Simpson and McFadden, 2005), these two principal components were picked to describe the underlying disparity sensitivity channels. In order to make sense of them, these two principal components, or factors, were then rotated using a varimax orthogonal rotation to obtain a simple structure accounting for the channel tuning curves (Kaiser, 1958; Peterzell and Teller, 2000; Simpson and McFadden, 2005; Peterzell, 2016). These factors-tuning curves are reported in Figure 3C. The first factor peaks at the highest measured frequency $2.4 \mathrm{c} / \mathrm{d}$ and the second peaks around $0.65 \mathrm{c} / \mathrm{d}$. They characterize the high and low spatial frequency channels identified by the inter-correlation analysis (respectively blue and green regions in Figure 2).

We wanted to test if the two channels we identified could in fact account for different classes within the population. In order to estimate the weights $\beta$ of each of these factors in each individual sensitivity, we projected our dataset onto the basis defined by the two identified factors. The best linear unbiased estimator of $\beta$ is obtained using the Moore-Penrose pseudo inverse $\mathrm{X}^{+}$(equation 1$)$ :

$$
\beta=\mathrm{X}^{+} \mathrm{y}
$$

where $\mathrm{y}$ is the matrix of all individual sensitivities, $\mathrm{X}^{+}$is the Moore-Penrose pseudo inverse of the new basis matrix $\mathrm{X}$ whose two columns represent the two factors and $\beta$ is a two-rows matrix in wihich each column contains the pair of weights associated to the two factors estimated for each subject (Friston et al., 1995; Woolrich et al., 2004; Reynaud et al., 2011).

The sensitivities $\hat{y}$ reconstructed solely from the linear combination of these two factors are plotted in Figure 4A 

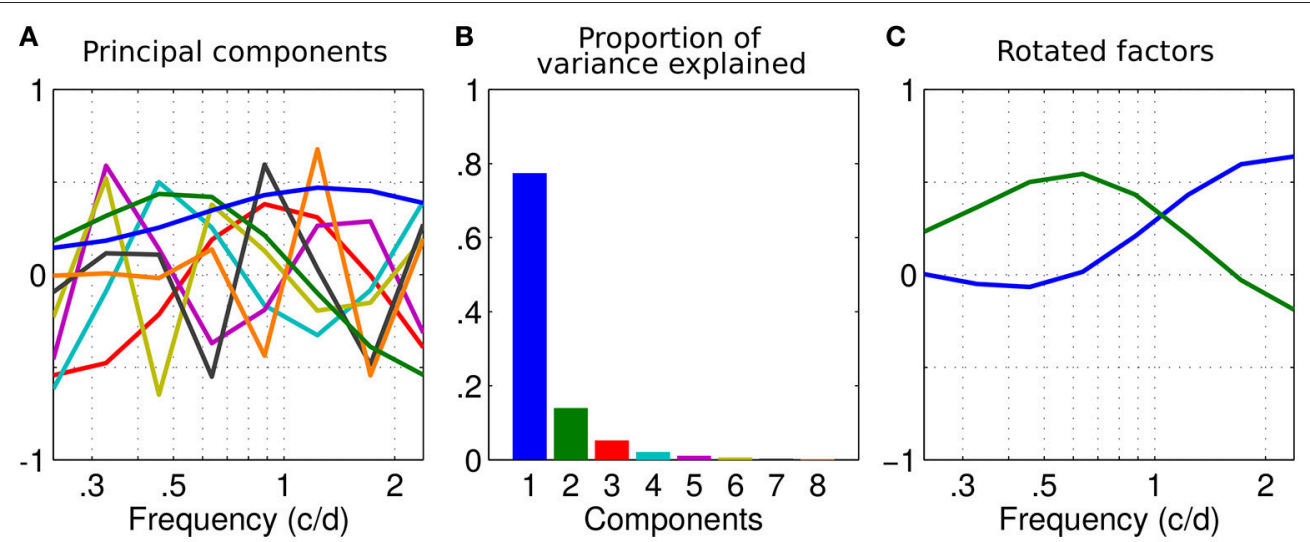

FIGURE 3 | Factor analysis. (A) Principal components of the dataset as a function of spatial frequency. Their order is indicated by colors in (B). (B) Scree plot of the variance explained by each component of the principal component analysis (PCA) in (A). (C) First two components rotated using a varimax rotation.
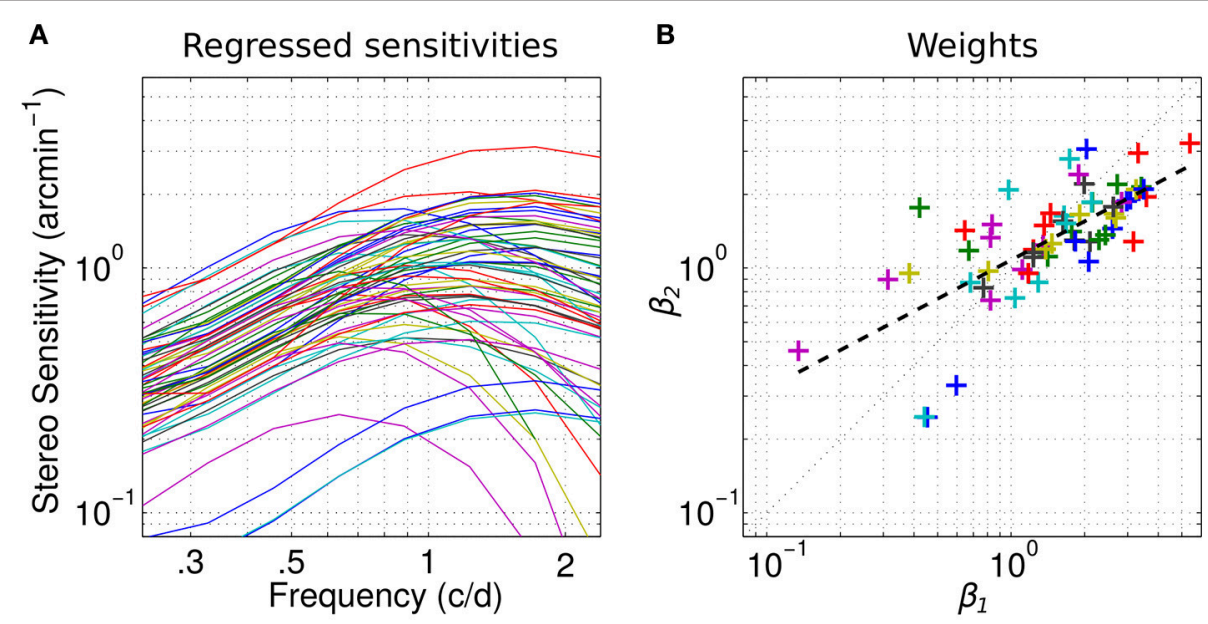

FIGURE 4 | Channels weights. (A) Individual sensitivities replotted using only the two channels factors. Same color-code as in Figure 1. (B) Scatterplot of the weights of the first factor $\beta_{1}$ vs. the weights of the second factor $\beta_{2}$ for all observers. Dashed line indicates linear regression on the log-values of the weights.

(Equation 2):

$$
\hat{y}=\mathrm{X} \beta
$$

We can see that they overall faithfully reproduce the original sensitivities except for the very low-pass profiles whose peaks shift to the right.

To determine whether these channels can account for different classes within the population, we report a scatterplot of the weights $\beta_{1}$ of the first factor vs. the weights $\beta_{2}$ of the second factor in Figure 4B for all observers. The mean weights for the first and second factor are, respectively, 1.76 and 1.48. As expected from the explained variance (Figure 3B), the weight of the first factor-the high-frequency channel-is greater than the weight of the second-the low frequency channel-in $70 \%$ of the cases. The distribution of these weights appears homogeneous and no clusters are revealed. However, the weights of the first factor seem to be relatively greater than the weights of the second in the high values range whereas it seems to be slightly the opposite in the low values range. This is further revealed by the slope of the linear regression between the log-values of the weights 0.53 , which is inferior to 1 (dashed line). In fact, the correlation between the weight is very high (coefficient of determination $R^{2}=0.51, p<$ 0.0001). Altogether, these observations suggest that the weight of the low and high spatial frequency channels co-vary: when the sensitivity is high for the low frequency channel, it is high for the high frequency channel too. But the high frequency channel contributes relatively more when the sensitivity is high and the low-frequency channel contributes relatively more when the sensitivity is low, in accordance with our previous observations (Reynaud et al., 2015).

\section{DISCUSSION}

The qDSF method assumes the sensitivity function follows the truncated log-parabola model and hence has a bell shape with a constant part, an increase to a peak and a drop-off 
(Watson and Robson, 1981; Lesmes et al., 2010). We previously showed that this model can accurately represent the sensitivity function compared to non-constrained methods (Reynaud et al., 2015) and documents large differences in sensitivities within the population (see Figure 1). For different individuals, this function can peak at very different frequencies and can show lowpass, band-pass or high-pass profiles. The resultant variability in sensitivity across spatial frequency provides a rich dataset for inter-correlation analyses (Peterzell et al., 1995; Peterzell, 2016).

Because two regions of inter correlations were identified among the population in Figure 1 and because 2 components accounted for more than $91 \%$ of the variance, our data could accurately be described by just 2 channels. However, the criterion to select the number of meaningful components in a PCA may vary. Popular selection methods such as a scree plot (Jackson, 1993) or the Random average under permutation analysis will indeed determine 2 components while some other methods will give less (the broken stick method gives 1 component) or more (the parallel analysis gives barely 3 , the kaiser Guttman criterion which recommends eigenvalues $>1$ gives 3 too). Some methods such as the Bartlett tests even recommends all the 8 components which would not reduce the dimensionality of the data (Bartlett, 1950). A complete description of these methods can be found in Peres-Neto et al. (2005).

Hence, we cannot completely rule out the possibility of a single-channel or multiple-channels hypothesis. Serrano-Pedraza and Read reported a single channel mechanism specific to vertical corrugations (Serrano-Pedraza and Read, 2010, though see Witz et al., 2014). However, the large difference we can observe between the lowpass profile of sensitivity for some observers compared to the bandpass of other ones would indicate that more than one channel are involved. Several studies suggested a multiple-channels mechanism (Julesz and Miller, 1975; Schumer and Ganz, 1979; Cobo-Lewis and Yeh, 1994; Glennerster and Parker, 1997; Serrano-Pedraza et al., 2013) with a broad channel tuning of $\sim 2-3$ octaves, comparable to our observations (Schumer and Ganz, 1979; Cobo-Lewis and Yeh, 1994). It is then possible that the 2 channels we observe are part of a multiple-channels system covering a wider range of spatial frequencies or could also overlap with intermediate channels continuously covering the spatial frequency range. Yang and Blake (1991) also observed two spatial frequency channels for disparity sensitivity using a masking paradigm. They described one channel centered around $3 \mathrm{c} / \mathrm{d}$ which could correspond to the high spatial frequency channel we observed and one centered around $5 \mathrm{c} / \mathrm{d}$. However, their study and the present study didn't measure the same spatial frequency range which might explain why they didn't identify our low spatial frequency channel and why we didn't observe their high one.

\section{REFERENCES}

Bartlett, M. S. (1950). Tests of significance in factor analysis. Br. J. Stat. Psychol. 3, 77-85.

Billock, V. A., and Harding, T. H. (1996). Evidence of spatial and temporal channels in the correlational structure of human spatiotemporal contrast sensitivity. J. Physiol. 490(Pt 2), 509-517.
The results of the present study suggests that there are two channels (Figure 4B), a low frequency channel that contributes to the detection of low corrugation frequencies and a more sensitive high frequency channel that contributes to the detection of high corrugation frequencies. We didn't observe any dichotomy based on these two channels within our population (Wilcox and Allison, 2009) which confirms the observations of most other population studies (Coutant and Westheimer, 1993; Bohr and Read, 2013; Bosten et al., 2015).

The implications of the assessment of the tuning of these disparity channels could be important in computer vision to design behaviorally relevant stereo matching algorithms. For instance, it could be used to tune the different layers of multiscale algorithms (Rohaly and Wilson, 1993) or provide fine and coarse scales for algorithms processing in center and periphery, respectively, as stereopsis could be mediated by different mechanisms in central and peripheral vision (Wardle et al., 2012; Witz and Hess, 2013).

\section{CONCLUSION}

The analysis of the inter-correlations in the disparity sensitivity as a function of the spatial frequency, revealed two disparity channels. With a factor analysis of the population data, we determined that the first channel is tuned to high spatial frequencies (peaks at $2.4 \mathrm{c} / \mathrm{d}$ ) and the second is tuned to low spatial frequencies (peaks at $0.65 \mathrm{c} / \mathrm{d}$ ). We also observed that these two channels are well correlated with each other. Our results confirm that disparity is encoded by multiple spatial frequency channels that are of different sensitivities and subserve different ranges of corrugation frequencies.

\section{AUTHOR CONTRIBUTIONS}

$\mathrm{AR}$ and $\mathrm{RH}$ designed the research and wrote the manuscript. AR analyzed the data.

\section{FUNDING}

This work was supported by a Natural Sciences and Engineering Research Council of Canada grant (NSERC \#46528) to RH.

\section{ACKNOWLEDGMENTS}

We thank the three reviewers for their helpful comments and suggestions. This work was supported by a Natural Sciences and Engineering Research Council of Canada grant (NSERC \#46528) to $\mathrm{RH}$.

Bohr, I., and Read, J. C. A. (2013). Stereoacuity with frisby and revised FD2 stereo tests. PLoS ONE 8:82999. doi: 10.1371/journal.pone. 0082999

Bosten, J. M., Goodbourn, P. T., Lawrance-Owen, A. J., Bargary, G., Hogg, R. E., and Mollon, J. D. (2015). A population study of binocular function. Vision Res. 110(Pt A), 34-50. doi: 10.1016/j.visres.2015. 02.017 
Cobo-Lewis, A. B., and Yeh, Y. Y. (1994). Selectivity of cyclopean masking for the spatial frequency of binocular disparity modulation. Vision Res. 34, 607-620. doi: 10.1016/0042-6989(94)90016-7

Coutant, B. E., and Westheimer, G. (1993). Population distribution of stereoscopic ability. Ophthalmic Physiol. Opt. 13, 3-7.

Friston, K., Holmes, A., Worsley, K., Poline, J., Frith, C., and Frackowiak, R. (1995). Statistical parametric maps in functional imaging: a general linear approach human brain. Mapping 2, 189-210. doi: 10.1111/j.1475-1313.1993.tb00419.x

Glennerster, A., and Parker, A. (1997). Computing stereo channels from masking data. Vision Res. 37, 2143-2152.

Jackson, D. A. (1993). Stopping rules in principal components analysis: a comparison of heuristical and statistical approaches. Ecology 74, 2204-2214. doi: $10.2307 / 1939574$

Julesz, B., and Miller, J. E. (1975). Independent spatial-frequency-tuned channels in binocular fusion and rivalry. Perception 4, 125-143. doi: 10.1068/p040125

Kaiser, H. F. (1958). The varimax criterion for analytic rotation in factor analysis. Psychometrika 23, 187-200.

Lesmes, L. A., Lu, Z.-L., Baek, J., and Albright, T. D. (2010). Bayesian adaptive estimation of the contrast sensitivity function: the quick CSF method. J. Vision 10, 17.1-17.21. doi: 10.1167/10.3.17

Lonini, L., Forestier, S., Teulière, C., Zhao, Y., Shi, B., and Triesch, J. (2013). Robust active binocular vision through intrinsically motivated learning. Front. Neurorobot. 7:20. doi: 10.3389/fnbot.2013.00020

Marr, D., and Poggio, T. (1979). A computational theory of human stereo vision. Proc. R. Soc. Lond. B Biol. Sci. 204, 301-328 doi: 10.1098/rspb.1979.0029

Mayer, M. J., Dougherty, R. F., and Hu, L.-T. (1995). A covariance structure analysis of flicker sensitivity. Vision Res. 35, 1575-1583.

Nishihara, H. K. (1984). Practical real-time imaging stereo matcher. Opt. Eng. SPIE. Int. Soc. Opt. Eng. 23.

Norcia, A. M., Sutter, E. E., and Tyler, C. W. (1985). Electrophysiological evidence for the existence of coarse and fine disparity mechanisms in human. Vision Res. 25, 1603-1611. doi: 10.1016/0042-6989(85)90130-0

Owsley, C., Sekuler, R., and Siemsen, D. (1983). Contrast sensitivity throughout adulthood. Vision Res. 23, 689-699. doi: 10.1016/0042-6989(83)90210-9

Peres-Neto, P. R., Jackson, D. A., and Somers, K. M. (2005). How many principal components? stopping rules for determining the number of non-trivial axes revisited. Comput. Stat. Data Anal. 49, 974-997. doi: 10.1016/j.csda.2004.06.015

Peterzell, D. H. (2016). Discovering sensory processes using individual differences: a review and factor analytic manifesto. Electron. Imaging Human Vision Electron. Imaging 11, 1-11. doi: 10.2352/ISSN.2470-1173.2016.16.HVEI-112

Peterzell, D. H., and Teller, D. Y. (1996). Individual differences in contrast sensitivity functions: the lowest spatial frequency channels. Vision Res. 36, 3077-3085. doi: 10.1016/0042-6989(96)00061-2

Peterzell, D. H., and Teller, D. Y. (2000). Spatial frequency tuned covariance channels for red-green and luminance-modulated gratings: psychophysical data from human adults. Vision Res. 40, 417-430. doi: 10.1016/S0042-6989(99)00187-X

Peterzell, D. H., Werner, J. S., and Kaplan, P. S. (1995). Individual differences in contrast sensitivity functions: longitudinal study of 4-, 6- and 8-month-old human infants. Vision Res. 35, 961-979. doi: 10.1016/0042-6989(94)00117-5

Piater, J. H., Grupen, R. A., and Ramamritham, K. (1999). "Learning realtime stereo vergence control," in Proceedings of the 1999 IEEE International Symposium on Intelligent Control Intelligent Systems and Semiotics (Cat. No. 99CH37014), (Cambridge, MA), 272-277.

Prince, S. J. D., Eagle, R. A., and Rogers, B. J. (1998). Contrast masking reveals spatial-frequency channels in stereopsis. Perception 27, 1345-1355. doi: $10.1068 / \mathrm{p} 271345$

Pulliam, K. (1982). "Spatial frequency analysis of three-dimensional vision," in Visual Simulation and Image Realism II, Proceedings of SPIE International Socity for Optical and Photonics, ed K. Setty, (San Diego, CA).

Quam, L. H. (1987). "Readings in Computer Vision: Issues, Problems, Principles, and Paradigms," in Hierarchical Warp Stereo, eds M. A. Fischler and O. Firschein (San Francisco, CA: Morgan Kaufmann Publishers Inc.), 80-86.

Read., J., Serrano-Pedraza, I., Widdall, M., and Peterzell, D. (2016). Sensitivity to horizontal and vertical sine-wave corrugations defined by binocular disparity: factor analysis of individual differences reveals discrete processes with broad orientation and spatial frequency tuning. J. Vision 16:833. doi: $10.1167 / 16.12 .833$
Reynaud, A., Gao, Y., and Hess, R. F. (2015). A normative dataset on human global stereopsis using the quick Disparity Sensitivity Function (qDSF). Vision Res. 113 (Pt A), 97-103. doi: 10.1016/j.visres.2015.04.021

Reynaud, A., Takerkart, S., Masson, G. S., and Chavane, F. (2011). Linear model decomposition for voltage-sensitive dye imaging signals: application in awake behaving monkey. Neuroimage 54, 1196-1210. doi: 10.1016/j.neuroimage.2010.08.041

Rohaly, A. M., and Wilson, H. R. (1993). Nature of coarse-to-fine constraints on binocular fusion. J. Opt. Soc. 10, 2433-2441. doi: 10.1364/JOSAA.10. 002433

Rosli, Y., Bedford, S. M., and Maddess, T. (2009). Low-spatial-frequency channels and the spatial frequency-doubling illusion. Invest. Opthalmol. Visual Sci. 50, 1956-1963. doi: 10.1167/iovs.08-1810

Schumer, R., and Ganz, L. (1979). Independent stereoscopic channels for different extents of spatial pooling. Vision Res. 19, 1303-1314. doi: 10.1016/0042-6989(79)90202-5

Sekuler, R., Wilson, H. R., and Owsley, C. (1984). Structural modeling of spatial vision. Vision Res. 24, 689-700.

Serrano-Pedraza, I., Brash, C., and Read, J. C. A. (2013). Testing the horizontalvertical stereo anisotropy with the critical-band masking paradigm J. Vision 13, 15-15. doi: 10.1167/13.11.15

Serrano-Pedraza, I., and Read, J. C. A. (2010). Multiple channels for horizontal, but only one for vertical corrugations? A new look at the stereo anisotropy. J. Vision 10:10. doi: 10.1167/10.12.10

Shioiri, S., Hatori, T., Yaguchi, H., and Kubo, S. (1994). Spatial frequency channels for stereoscopic depth perception. Opt. Rev. 1, 311-313.

Simpson, W. A., and McFadden, S. M. (2005). Spatial frequency channels derived from individual differences. Vision Res. 45, 2723-2727. doi: 10.1016/j.visres.2005.01.015

Tsai, R. Y. (1986). "An efficient and accurate camera calibration technique for 3d machine vision," in Proceedings of IEEE Conference on Computer Vision and Pattern Recognition (Miami Beach, FL), 364-374.

Tyler, C. W., Barghout, L., and Kontsevich, L. L. (1994). "Computational reconstruction of the mechanisms of human stereopsis," in Computational Vision Based on Neurobiology, SPIE International Society Optical Engineering, ed T. B. Lawton, (Park Grove, CA).

Wardle, S. G., Bex, P. J., Cass, J., and Alais, D. (2012). Stereoacuity in the periphery is limited by internal noise. J. Vision 12:12. doi: 10.1167/12.6.12

Watson, A. B., and Robson, J. G. (1981). Discrimination at threshold: labelled detectors in human vision. Vision Res. 21, 1115-1122. doi: 10.1016/0042-6989(81)90014-6

Wilcox, L. M., and Allison, R. S. (2009). Coarse-fine dichotomies in human stereopsis. Vision Res. 49, 2653-2665. doi: 10.1016/j.visres.2009 .06 .004

Witz, N., and Hess, R. F. (2013). Mechanisms underlying global stereopsis in fovea and periphery. Vision Res. 87, 10-21. doi: 10.1016/j.visres.2013. 05.003

Witz, N., Zhou, J., and Hess, R. F. (2014). Similar mechanisms underlie the detection of horizontal and vertical disparity corrugations. PLoS ONE 9:e84846. doi: 10.1371/journal.pone.0084846

Woolrich, M. W., Behrens, T. E. J., and Smith, S. M. (2004). Constrained linear basis sets for HRF modelling using Variational Bayes. Neuroimage 21, 1748-1761. doi: 10.1016/j.neuroimage.2003.12.024

Yang, Y., and Blake, R. (1991). Spatial frequency tuning of human stereopsis. Vision Res. 31, 1177-1189 doi: 10.1016/j.neuroimage.2003. 12.024

Conflict of Interest Statement: The authors declare that the research was conducted in the absence of any commercial or financial relationships that could be construed as a potential conflict of interest.

Copyright (c) 2017 Reynaud and Hess. This is an open-access article distributed under the terms of the Creative Commons Attribution License (CC BY). The use, distribution or reproduction in other forums is permitted, provided the original author(s) or licensor are credited and that the original publication in this journal is cited, in accordance with accepted academic practice. No use, distribution or reproduction is permitted which does not comply with these terms. 